\title{
Modelo dinámico de las afectaciones del tiempo de ciclo kanban en el flujo de producción de una línea de ensamble
}

\author{
Lisaura Walkiria Rodriguez Alvarado, Jesús Loyo Quijada, \\ Miguel Ángel López Ontiveros \\ Universidad Autónoma Metropolitana, UAM-Azcapotzalco, Ciudad de México, México \\ \{Lwra, lqj, mlopez\}@correo.azc.uam.mx
}

\begin{abstract}
Resumen. En este artículo se presenta el modelo dinámico del proceso de ensamble de reductores de velocidad como parte de una actividad realizada en un taller de simulación kanban dirigido a alumnos de ingeniería industrial. El principal objetivo del modelo desarrollado es analizar el comportamiento de los niveles de inventario de las estaciones de trabajo y las acumulaciones de las tarjetas kanban (ordenes de producción) derivado de las afectaciones del tiempo de ciclo kanban en los flujos de producción. Los resultados del análisis y evaluación muestran que, cuando el tiempo de ciclo kanban es superior al tiempo de entrega del lote de producción entre estaciones, se genera una acumulación de órdenes de producción. Esto ocasiona que una vez que el flujo de producción alcanza su máximo rendimiento este disminuya. Esa situación provoca que los niveles de inventario en proceso no correspondan al ritmo del cliente, generando órdenes de producción sin entregar a tiempo.
\end{abstract}

Palabras claves: Dinámica de sistemas, flujo de producción, sistema kanban.

\section{Dynamic Model of Kanban Cycle Time Implications in the Production Flow of an Assembly Line}

\begin{abstract}
This article describes the dynamic model of assembly process of reducer is presented as part of an activity performed in a workshop kanban simulation aimed at students of industrial engineering. The main objective of the model developed is to analyze the behavior of inventory levels workstations and accumulations of kanban cards (production orders) derived from the effects of the cycle time kanban in production flows. The results of analysis and evaluation show that when kanban cycle time is longer than the time of delivery of batch production between stations, an accumulation of production orders is generated. This causes once that the production flow reaches its maximum performance, this
\end{abstract}


decrease. This situation causes the WIP levels do not correspond to the rhythm to the client, generating production orders not deliver on time.

Keywords: System dynamics, production flow, kanban system

\section{Introducción}

El concepto de Manufactura Esbelta (ME) evolucionó a partir de la industria Japonesa, específicamente del Sistema de Producción Toyota (SPT). En [1] la ME es considerada como una filosofía que busca maximizar el valor agregado del producto al reducir o eliminar los desperdicios involucrados en el proceso de producción, mediante la aplicación de una serie de técnicas y metodologías fundamentadas en el SPT. Una de estas técnicas es el sistema kanban usada para la reducción, control de inventarios y el manejo de producción "Justo a Tiempo" (JIT) [2].

Lage y Godinho [3] conducen en su estudio, una revisión en la literatura para identificar, clasificar y analizar las variaciones del sistema kanban propuestos por diferentes investigadores como parte de aplicación de otras metodologías de mejora continua. En su estudio evidencian las dificultades de adaptación del sistema kanban a los nuevos requerimientos productivos El entorno de modelado y simulación ofrece un potencial de gran aplicación en la actualidad para analizar el comportamiento de estos sistemas de producción [4].

En lo que respecta al análisis de modelado y simulación de sistemas kanban utilizando la metodología de Dinámica de Sistemas (DS) se han desarrollado interesantes trabajos en aplicaciones prácticas y planteamientos teóricos [5, 6].

La mayoría de estos trabajos están enfocados en el manejo y control de una línea de producción sencilla por medio de tarjetas de retiro y producción, así como el nivel de respuesta que presenta el sistema ante condiciones de incertidumbre $[7,8,9,10,11$, 12]. Por otro lado diferentes investigaciones consideran analizar el desempeño del sistema Kanban en una línea de producción con variedad de productos [13, 14, 15], y tomar en cuenta los efectos de diferentes factores operacionales en el desempeño del sistema productivo controlado por tarjetas kanban $[16,17,18,19]$.

En aplicaciones recientes se ha considerado emplear el sistema kanban en conjunto con otros sistemas control conocidos como sistemas Híbridos. [20, 21, 22, 23]. En este trabajo se presenta un modelo dinámico desarrollado a partir de una actividad didáctica de un taller de simulación kanban dirigido a alumnos de ingeniería industrial. El modelo dinámico está enfocado al análisis de los efectos del tiempo del ciclo kanban en el flujo de producción de una línea de ensamble de reductores de velocidad, en el cual se ejemplifica el comportamiento real de la aplicación del sistema kanban en un ejercicio simulado con los alumnos. El modelo permite explorar el comportamiento de la línea de ensamble ante el efecto de las incidencias ocasionadas por las variaciones en el tiempo de ciclo kanban, tomando como parámetros de análisis el inventario en proceso, tiempo de entrega y acumulación de órdenes de producción. 


\section{Funcionamiento del sistema kanban}

El sistema kanban es definido como un mecanismo de control para el flujo de material [24] mediante la utilización de tarjetas de producción y retiro de producto, las cuales garantizan la producción de la cantidad adecuada en el momento adecuado. Este sistema permite que cada estación de trabajo opere con los niveles mínimos de inventario, al procesar únicamente lo solicitado por la estación siguiente [25, 26].

La explicación del funcionamiento del sistema kanban se explica con ayuda de la Fig. 1. La producción de la última estación de la línea de ensamble, en este caso montaje, es activada una vez que el cliente realiza una solicitud. En ese momento se toma material de esa estación para cubrir el requerimiento del cliente, al mismo tiempo se activa una tarjeta kanban (orden de producción), la cual en función con los niveles de inventario de la estación montaje, las estaciones engrane, cárter, corona A y corona B y las órdenes del cliente, activa la cantidad a cubrir de acuerdo a lo requerido por la estación montaje, lo cual debe ser igual a lo solicitado por el cliente. Esto ocurre cada cierto tiempo determinado por el tiempo de ciclo kanban ${ }^{1}$,

\section{Desarrollo del modelo dinámico}

\subsection{Entorno de simulación}

El modelo desarrollado es una representación de lo que ocurre realmente durante el taller de simulación kanban dirigido a alumnos de ingeniería industrial. En el taller los estudiantes comprenden los mecanismos claves en la gestión de la producción mediante la interacción y juego de roles desempeñado por ellos mismos.

(a)

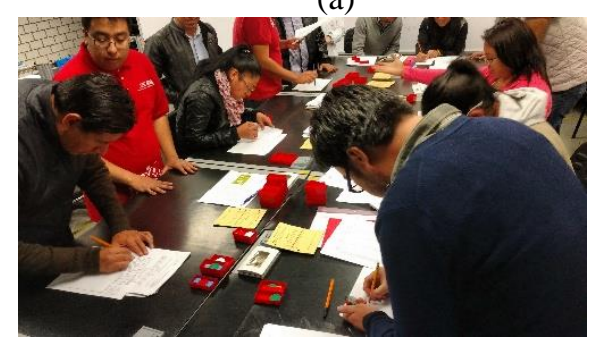

(b)

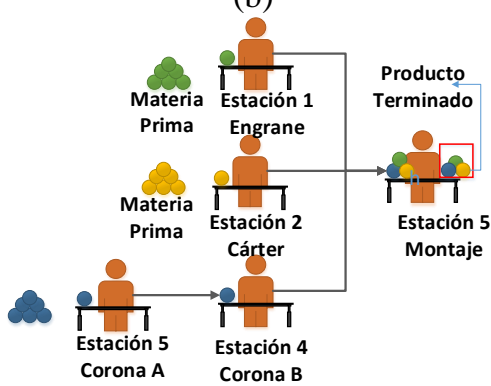

Fig. 1. (a) Taller de simulación kanban. (b) Proceso de ensamble del reductor.

Como parte de la didáctica del taller se establece una línea de ensamble de 4 estaciones de trabajo para fabricar los componentes que componen los reductores de velocidad. Cada componente en cada estación de trabajo se fabrica a partir de materia prima tomada en el stock de las estaciones de engrane, cárter, corona A y corona B (en

${ }^{1}$ El tiempo interno desde que la tarjeta kanban es tomada de la caja de colección 
este caso, la estación corona A surte a estación corona B). Posteriormente se reúnen los componentes semiprocesados en la estación montaje para realizar el ensamble del producto final.

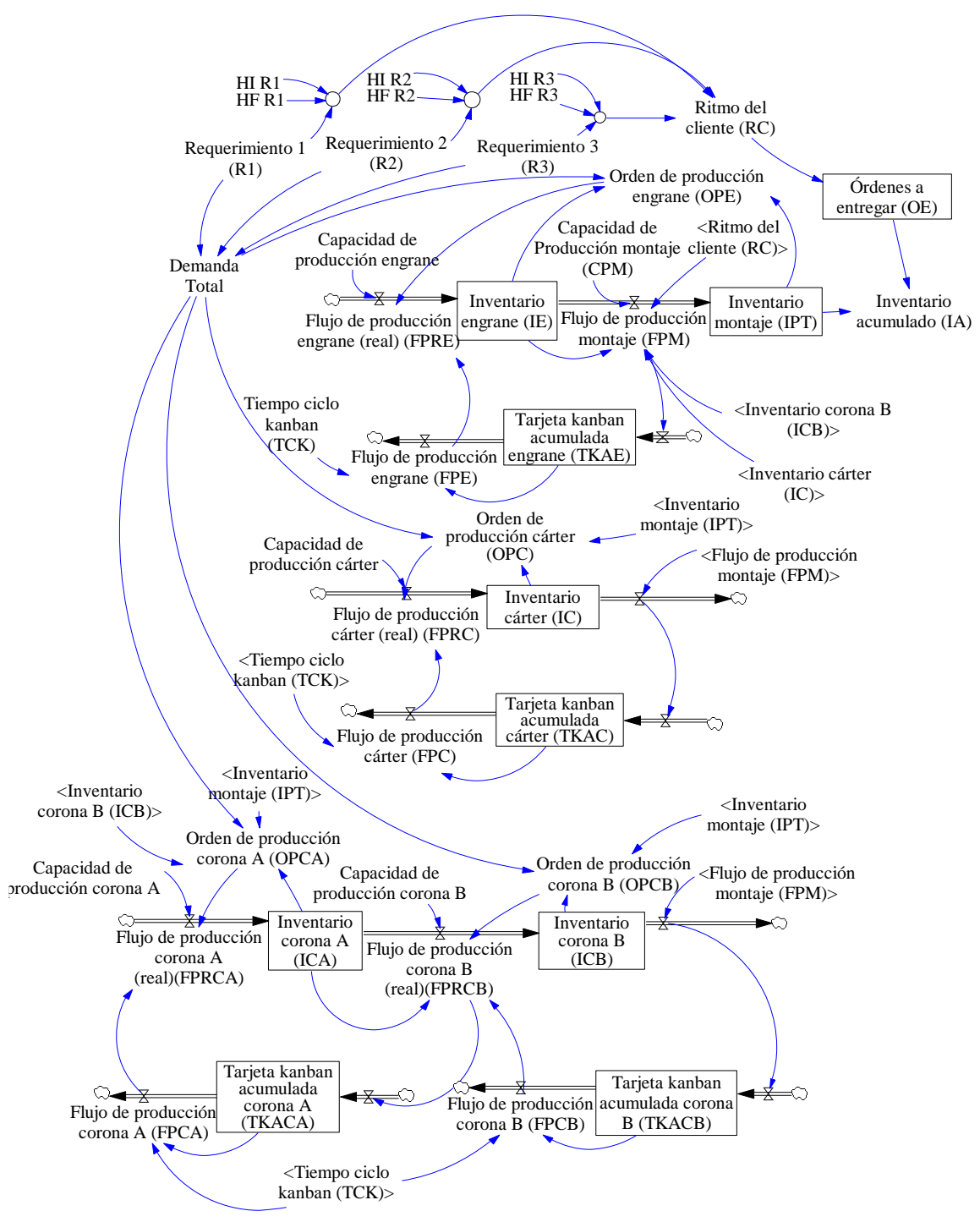

Fig. 2. Modelo dinámica de la línea de ensamble de un reductor.

\subsection{Estructura del modelo}

En Fig. 2 se presenta el modelo desarrollado, el cual permite analizar el comportamiento dinámico del flujo de producción de las estaciones engrane, cárter, 
corona A, corona B y montaje, controladas por las tarjetas kanban. Se toma como principal referencia de análisis para el modelo la acumulación de los niveles de inventario, acumulación de tarjetas kanban, su respectivo tiempo de ciclo y las órdenes de producción pendientes a entregar. La interrelación de estas variables determina la secuencia productiva de cada estación controlada por las tarjetas Kanban.

El análisis de simulación inicia una vez que el cliente establece la Demanda Total (comprendida en este caso por tres requerimientos en una jornada laboral). El Ritmo del cliente es quien determina la velocidad del flujo de producción de la última estación en el proceso de ensamble del reductor, en este caso corresponde a la estación Montaje. Cuando se presenta una diferencia entre el comportamiento del flujo de producción de la estación montaje, (determinada por su capacidad y el ritmo del cliente) y el tiempo de reacción de cada estación predecesora (determinado por el tiempo de ciclo kanban), ocasiona una acumulación de Tarjetas Kanban. De esta forma, una estación produce únicamente como respuesta a una orden de reposición inmediatamente posterior de productos consumidos por la estación montaje.

Estas características determinan el Flujo de producción real para cada estación, el cual se ve restringido por la Capacidad de producción y las Órdenes de Producción a cumplir. Por otro lado, cada Orden de producción para cada estación, se determina por la Demanda Total establecida por el cliente y por los niveles de Inventario en proceso de cada componente, a mayor nivel de inventario existentes, las órdenes de producción son menores. Bajo estas consideraciones, en el comportamiento del proceso productivo se pueden presentar dos situaciones: La primera es que la velocidad de acumulación del inventario de producto terminado sea menor al ritmo establecido por el cliente ocasionado por las restricciones de capacidad de la misma línea. Y la segunda situación es que el sistema de control por medio de las tarjetas Kanban garantice que la velocidad de acumulación del inventario de producto terminado sea igual al ritmo establecido por el cliente.

\subsection{Conceptualización de las variables del sistema}

Para poder analizar y representar el comportamiento dinámico del proceso de ensamble de los reductores de velocidad en las diferentes estaciones de ensamble se consideraron las siguientes variables principales.

Ritmo del cliente (RC). Establece la velocidad con la cual el cliente solicita una determinada cantidad de producto, Requerimiento $o_{\mathrm{i}},\left(R_{i}\right)$. Para determinar el $R C$ se toma en consideración el tiempo disponible que tienen la línea de ensamble $\left(H I_{i}\right.$ y $\left.H F_{i}\right)$ para dar respuesta a dicha solicitad y la cantidad demandada en dicho periodo. En la ecuación 1 se presenta la función "step" ${ }^{2}$ ", sus dos argumentos determinan el punto de inicio y el tiempo en que tarda en completarse el requerimiento solicitado. Por otro lado, la sumatoria de los requerimientos determina la Demanda Total (DT).

\footnotetext{
${ }^{2}$ Esta función permite que los datos tengan un comportamiento como entrada a escalón. El valor en esta función tiene un punto de inicio, se mantiene constante y en un punto final vuelve a su condición inicial. Los puntos de inicio y finalización están determinados por HF y HI:
} 
Lisaura Walkiria Rodriguez Alvarado, Jesús Loyo Quijada, Miguel Ángel López Ontiveros

$$
\mathrm{Rc}=\sum^{\mathrm{i}=\mathbf{1}}\left(\left(\operatorname{Step}\left(\left(\mathrm{R}_{\mathrm{i}} /\left(\mathrm{HFR}_{\mathrm{i}}-\mathrm{HIR}_{\mathrm{i}}\right)\right), \mathrm{HIR}_{\mathrm{i}}\right)-\mathrm{Step}\left(\left(\mathrm{R}_{\mathrm{i}} /\left(\mathrm{HFR}_{\mathrm{i}}-\mathrm{HIR}_{\mathrm{i}}\right)\right), \mathrm{HFR}_{\mathrm{i}}\right)\right) .\right.
$$

Flujos de producción. Determinan la velocidad de producción de cada una de las estaciones para el ensamble del reductor.

- Flujo de producción montaje $(F P M)$. La velocidad del flujo de producción de esta estación está condicionada por la cantidad de inventario mínimo disponibles de producto semiensamblado de las estaciones anteriores (IE inventario engrane, IC inventario cárter, ICA inventario corona A, ICB inventario corona B), la capacidad de producción de la estación montaje $(C P M)$ y el $R C$. La ecuación 2 establece esta relación:

$$
\text { FPM=Min (Min (IE, IC, ICB), (CPM, RC)). }
$$

- Flujo de producción de las demás estaciones de trabajo (FPE, FPC, FPCA, FPCB). Estos flujos están determinados por la acumulación de tarjetas kanban $(T K A)$ en cada estación y su respectivo tiempo de entrega establecido por el tiempo de ciclo kanban $(T C K)$. Donde, la acumulación de las tarjetas se pueden representar como un inventario en proceso de acuerdo con la Ley de Little, la cual establece que, la tasa de producción $=$ inventario en proceso/ tiempo de entrega, esta relación se presenta en ecuación 3:

$$
\text { FPestación=TKAestación/TCK. }
$$

- Flujo de producción real de las demás estaciones de trabajo (FPRE, FPRC, FPRCA, $F P R C B)$. Estos flujos están condicionados por las órdenes de producción a cumplir $(O P)$, capacidad de producción $(C P)$ y por el flujo de producción de las estaciones de trabajo derivado de la relación entre el número de tarjetas kanban y tiempo ciclo. En el caso del FPRCB tiene una restricción adicional correspondiente al nivel de inventario de Corona A. A pesar de la acumulación de las tarjetas Kanban las estaciones de trabajo no pueden producir más de lo establecido por las $O P$ y su restricción de $C P$ esta relación se presenta en ecuación 4:

FPRestación=If then else (OPestación $\leq 0,0$, Min (CPestación, FPestación)).

Orden de producción (OPestación). La $O P$ de cada estación establece la cantidad máxima a producir, la cual está determinada por la diferencia de la $D T$ establecida por el cliente y los niveles de inventarios de las estaciones siguientes y los niveles de su propia estación. La ecuación 5 describe el comportamiento de dicha variable:

OPestación=DT-Iestación actual-Iestación siguiente.

Niveles de inventario. Determinan las acumulaciones de inventario en proceso y producto terminado.

- Inventario de producto terminado (IPT). Representa la acumulación de material procesado procedente de la estación montaje, su expresión está representada por la ecuación 6: 
Modelo dinámico de las afectaciones del tiempo de ciclo kanban en el flujo de producción ...

$$
\operatorname{IPT}(\mathrm{t})=\operatorname{FPM}(\mathrm{t}) \text {. }
$$

- Inventario de las demás estaciones (IE, IC, ICA, ICB). Representa la acumulación de material semiprocesado procedente de las demás estaciones de trabajo anteriores a la estación montaje. Su comportamiento está determinada en ecuación 7 , establecida por la diferencia de flujos de producción de la estación anterior y la estación montaje

$$
\text { Iestación }(\mathrm{t})=\text { FPestación }(\mathrm{t}) \text {-FPM }(\mathrm{t})
$$

- Tarjeta kanban acumulada (TKA). Establece la acumulación de las tarjetas Kanban procedentes de estación montaje o estación corona B a lo largo del tiempo. Este nivel de acumulación se presenta debido a la diferencias de los flujos de producción de estas estaciones y el flujo de producción de la estación anterior. La ecuación 8 representa el comportamiento de dicha acumulación:

$$
\text { TKAestación (t)=FPM (t)-FPestación (t). }
$$

- Inventario Acumulado (IA). Establece la diferencia entre los niveles de inventarios procedentes de la estación montaje y las órdenes a entregar $(O E)$ determinadas por el ritmo del cliente, la ecuación 9 establece su comportamiento. Los inventarios iniciales de cada estación se balancean considerando el ritmo del cliente por el tiempo de entrega, así si el cliente solicita 100 unidades en la primera hora y el tiempo de entrega de la estación montaje es de una hora, el inventario inicial es igual a 100 Uds:

$$
\operatorname{IA}(\mathrm{t})=\operatorname{IPT}(\mathrm{t})-\mathrm{OE}(\mathrm{t})
$$

\section{Simulación y evaluación}

Para analizar el comportamiento dinámico del modelo desarrollado se recolectaron datos derivados de la actividad didáctica del taller de simulación kanban. Los participantes en sus diferentes roles de analistas y operadores, registran las características de los requerimientos del cliente a cumplir (R1, R2, R3), asi como el tiempo de entrega del producto final. Los resultados de las corridas de simulación en el taller permitieron obtener los datos que alimentan el modelo dinámico, desarrollado en el software Vensim Ple, para esto se consideraron dos escenarios de análisis. En el primer escenario se considera un tiempo de ciclo kanban (TCK) igual al tiempo de entrega de tamaño de lote de las estaciones de trabajo (1 hora). En un segundo escenario, se considera un tiempo de ciclo kanban igual a dos horas. Los valores de los parámetros y condiciones iniciales seleccionadas y secuencia de producción para verificar la validez de las ecuaciones y la veracidad de los resultados obtenidos, se muestran en Tabla 1.

El tiempo de ciclo kanban afecta directamente el comportamiento del flujo de producción de las estaciones anteriores a la estación montaje, ya que determina el tiempo de solicitud real de cada orden de producción. Esto ocasiona que los niveles de 
inventario en proceso varíen de acuerdo a este comportamiento incrementando o disminuyendo de acuerdo al ritmo establecido por el incremento de las tarjetas kanban (ecuación 7).

Tabla 1. Parámetros y condiciones iniciales.

\begin{tabular}{lcc}
\hline \multicolumn{1}{c}{ Parámetro } & Valor & Unidad \\
\hline Capacidad de producción de engrane $(C P E)$ & 100 & Uds./hora \\
Capacidad de producción de cárter $(C P C)$ & 100 & Uds./hora \\
Capacidad de producción de corona B $(C P C B)$ & 200 & Uds./hora \\
Capacidad de producción de corona A $(C P C A)$ & 200 & Uds./hora \\
Capacidad de producción de montaje $(C P M)$ & 100 & Uds./hora \\
Tiempo de ciclo kanban (TCK) primer escenario & 1 & hora \\
Tiempo de ciclo kanban (TCK) segundo escenario & 2 & horas \\
Requerimiento R1 & 100 & Uds. \\
Requerimiento R2 & 500 & Uds. \\
Requerimiento R3 & 200 & Uds. \\
Hora de inicio R1 $\left(\mathrm{HI}_{1}\right)$ y Hora de Finalización R1 $\left(\mathrm{HF}_{1}\right)$ & $0-1$ & Hora \\
Hora de inicio R2 $\left(\mathrm{HI}_{2}\right)$ y Hora de Finalización R2 $\left(\mathrm{HF}_{2}\right)$ & $2-6$ & Hora \\
Hora de inicio R3 $\left(\mathrm{HI}_{3}\right)$ y Hora de Finalización R3 $\left(\mathrm{HF}_{3}\right)$ & $6-8$ & Hora \\
\hline
\end{tabular}

(a)

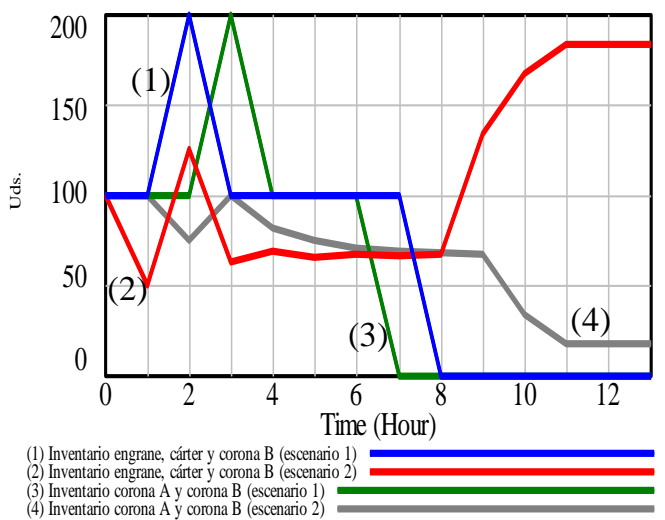

(b)

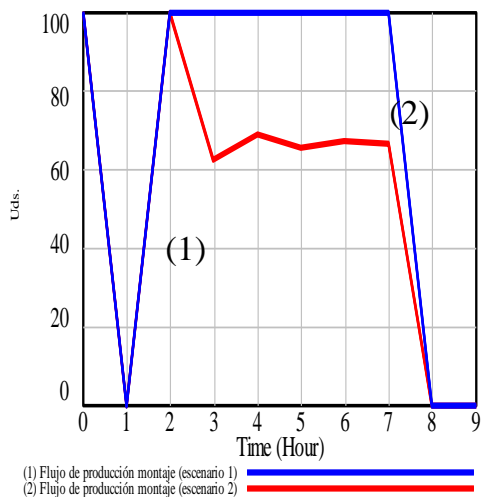

Fig. 2. (a) Comportamiento dinámico de los niveles de inventario en estaciones de trabajo (engrane, cárter, corona A, corona B) escenario 1 y 2. (b) Flujo de producción en estación Montaje.

En Fig. 2 (a) se puede observar que, cuando el tiempo de ciclo kanban es igual a 1 hora los niveles de inventario de engrane, cárter, corona A y corona B se mantienen constantes a un nivel de 100 unidades, con un incremento a 200 unidades desde la hora 1 y 2 hasta la hora 3 y 4 . Esto indica que los niveles de inventario de estas estaciones mantienen un nivel mínimo correspondiente al ritmo de producción establecido por el cliente. Por otro lado, al analizar el comportamiento de los niveles de inventario de 
estas mismas estaciones cuando el tiempo de ciclo kanban es de 2 horas se observa que dichos niveles se mantienen por debajo de las 100 unidades. Esto indica que los flujos de producción no están produciendo a la máxima capacidad permitida ni al ritmo del cliente establecido, esto se debe a que las órdenes procedentes de las tarjetas kanban presentan un retraso en su tiempo de entrega. Este retraso se transmite a cada flujo de producción de las estaciones engrane, cárter, corona A y B, por lo que su ritmo de producción es inferior al ritmo solicitado por el cliente. Los flujos de producción de estas estaciones, fluctúan de acuerdo al comportamiento de la acumulación de las tarjetas kanban, lo cual se ve reflejado en la acumulación de sus niveles de inventario. En Fig. 2 (b) se ejemplifica este comportamiento con el flujo de producción de estación Montaje para ambos escenarios. Es notorio observar las fluctuaciones del flujo productivo de la estación montaje cuando el tiempo de ciclo kanban es igual a 2 horas. Este comportamiento se debe al retraso ocasionado por el tiempo de entrega de las tarjetas kanban, lo que ocasiona que el flujo productivo de las estaciones predecesoras esté por debajo de la capacidad máxima permitida. Ocurre un caso totalmente diferente cuando el tiempo de ciclo kanban es igual a 1 hora, ya que el flujo productivo de la estación montaje se mantiene estable a lo largo de la jornada laboral, sin interrupciones y trabajando a la capacidad máxima permitida. Esto garantiza que se de un cumplimiento efectivo de las órdenes de producción.

(c)

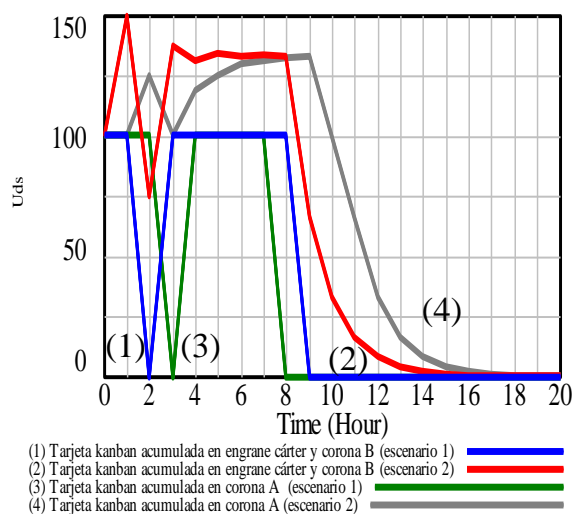

(d)

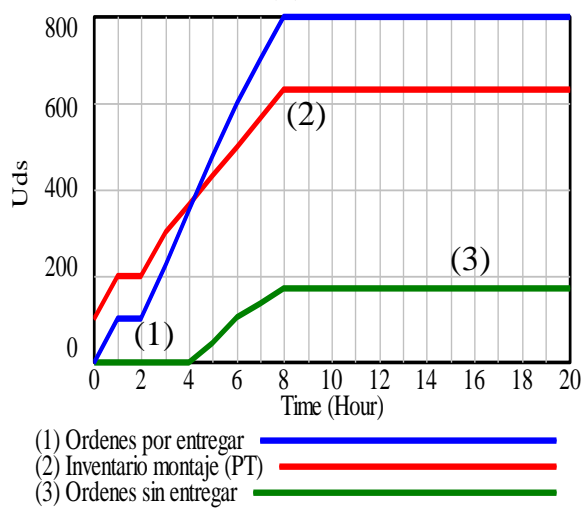

Fig. 3. (c) Acumulación de tarjetas kanban en las estaciones de trabajo. (d) Órdenes pendientes por entregar.

La acumulación de las tarjetas kanban indican las órdenes de producción en cada estación de trabajo. Su nivel de acumulación responde a la diferencia del flujo de producción montaje y el flujo de producción de cada estación anterior a esta. En Fig. 3 (a) se observa que, los niveles de inventario de las tarjetas kanban o bien, la cantidad de ordenes acumuladas cuando el tiempo de ciclo kanban es igual a 1, se mantienen relativamente constantes, igual a 100 unidades lo que corresponde al ritmo establecido por el cliente. Por otro lado, cuando el tiempo de ciclo kanban incrementa a dos horas, es evidente el incremento de las ordenes de producción para dichas estaciones, alcanzando niveles de 130 unidades a producir por hora. Este comportamiento 
sobrepasa directamente la capacidad de producción establecida por las estaciones de trabajo, por lo que los flujos de producción únicamente procesarán lo permitido de acuerdo a sus restricciones de capacidad. Esta situación ocasiona que se generen órdenes sin entregar a tiempo. En la Figura 3(d) se presenta que las órdenes sin entregan se presentan a partir de la hora 4 y continúan acumulándose hasta la hora 8 con un total de 180 unidades. Esta situación se presenta a partir de que el inventario en la estación montaje no alcanza los niveles máximos de 800 unidades debido al retraso generado por el tiempo de resurtimiento de las tarjetas kanban entre estaciones lo que ocasiona que no trabajen a su máxima capacidad, por lo tanto la estación montaje no tiene suficiente material para cumplir con las órdenes por entregar solicitadas por el cliente. Este comportamiento responde a la diferencia de los flujos de producción de las estaciones de trabajo afectadas por la variación en los tiempo de ciclo kanban, lo que ocasiona que las órdenes de producción no sean entregas a tiempo.

\section{Conclusiones}

La DS permite crear diferentes escenarios para analizar el comportamiento de sistemas productivos bajo diferentes consideraciones. En este caso de estudio se determinan las afectaciones de las variaciones del tiempo de ciclo kanban en una línea de ensamble de reductores, como parte de un ejercicio de simulación para analizar el comportamiento del sistema controlado por tarjetas kanban. Para esto, se analizaron dos escenarios comparativos de las variaciones del tiempo de ciclo kanban. En el primer escenario se consideró que el tiempo de ciclo kanban es igual a 1 hora, aquí se presenta que, los niveles de inventario entre las estaciones involucradas en el proceso mantendrán siempre un nivel máximo permitido (generalmente, es el nivel de producción en una hora de producción), para este caso, para las estaciones Engrane y cárter es igual a 100 unidades, y para las estaciones Corona A y B es igual a 200 unidades. Al mantener un flujo productivo igual al ritmo de producción del cliente y niveles de inventarios mínimos, se garantiza que el flujo de producción de la estación montaje sea estable y se complete las órdenes de producción, bajo consideración de su máxima capacidad productiva. Por otro lado, en el segundo escenario se consideró que el tiempo del ciclo kanban es de 2 horas. Para este caso se evidencia que, se genera una acumulación de órdenes de producción, las cuales son completadas en cada estación de acuerdo a su máxima capacidad permitida. Esto ocasiona que los flujos de producción de las estaciones trabajen a un rendimiento menor al permitido ya que se ven afectado por el retraso en la entrega de las tarjetas kanban que a su vez determinan las órdenes de producción a completar. Por lo tanto los niveles de inventario de producto en proceso en cada estación no corresponden al ritmo establecido por el cliente lo que de igual forma ocasiona acumulación de ordenes pendientes. Para contrarrestar esta situación y satisfacer el creciente número de órdenes de producción se debe incrementar el rendimiento o la capacidad del flujo de producción de las estaciones antecesoras a la estación montaje, ya que una vez que las estaciones alcanzan su máximo rendimiento no se registra ninguna mejora, lo que da paso a que se genere una acumulación de las órdenes sin entregar a tiempo. El análisis de las situaciones presentadas en ambos 
escenarios permite una válida representación de la situación real expuesta en el taller de simulación del Sistema kanban. Esto permitió que los alumnos comprendieran la importancia de determinar correctamente el tiempo de ciclo kanban y sus afectaciones en el sistema productivo.

\section{Referencias}

1. Sundar, R., Balaji, A. N., Satheesh Kumar, R. M.: A Review on Lean Manufacturing Implementation Techniques. Procedia Engineering 97, pp. 1875-1885 (2014)

2. Abdul, N. A., Mohd, S., Mohamed, M.: Lean Manufacturing Case Study with Kanban System Implementation. Procedia Economics and Finance 7, pp. 171-180 (2013)

3. Lage, M., Godinho, M.: Variations of the Kanban System: Literature Review and Classification. Int. J. Production Economics 125, pp. 13-21. (2010)

4. Mohsen, J., Tillal, E., Aisha, N., Lampros, S., Terry, Y.: Simulation in manufacturing and business: A review. European Journal of Operational Research vol. 203, pp. 1-13 (2010)

5. Tregubov, A., Lane, A.: Simulation of Kanban based scheduling for Systems: initial results. Procedia Computer Science 44, pp. 224-233 (2015)

6. Halim, N., Yusuf, N., Jaafar, R., Jaffar, A., Kaseh, A., Azira, N.: Effective Material Handling System for JIT Automotive Production Line. Procedia Manufacturing 2, pp. 251-257 (2015)

7. O’Callaghan, R.: A System Dynamics Perspective on JIT-Kanban. In: International Conference of the System Dynamics Society, pp. 959-1004 (1986)

8. Patel, K., Thanki, S. J.: System Dynamics Modelling an Analysis of a Single Stage Single Product Kanban Production System. International Journal of Innovative Research in Science, Engineering and Technology, Vol. 2, pp. 2262-2270 (2013)

9. Ebrahimpour, M., Fathi, B. M.: Dynamic Simulation of a Kanban Production Inventory System. International Journal of Operations \& Production Management. Bradford: Vol. 5, Issue 1, pp. 5-10 (1985)

10. Guerra, L., Murino, T., Romano, A.: A System Dynamics Model for a Single-Stage MultiProduct Kanban Production System. Recent Advances in Automation \& Information, pp. 171-176 (2010)

11. Ahmad, N., Ahmed, B., Noriah, B., Nurul H.: Implementation of Just in Time Production through kanban System. Industrial Engineering Letters, vol. 3, No. 6, pp. 11-20 (2013)

12. Lummus, R.: A simulation analysis of sequencing alternatives for JIT lines using kanbans. Journal of Operations Management, vol. 13, pp. 183-191 (1995)

13. Chan, F.T.S.: Effect of kanban size on just-in-time manufacturing systems. Journal of Materials Processing Technology, 116, pp. 146-160 (2001)

14. Murino, T., Naviglio, G., Romano, E., Zoppoli, P.: Single stage multi product kanban system. Optimization and parametric analysis. In: Proceedings of the 8th WSEAS International Conference on System Science and Simulation in Engineering, pp. 313- 318 (2009)

15. Mehmet, S., Abdullah Al-J.: Simulation analysis of just-in-time production system. Int. J. Production Economics, vol. 42, pp. 67-78 (1995)

16. Matta, A., Dallery, Y., Mascolo M.: Analysis of assembly systems controlled with kanbans. European Journal of Operational Research, vol. 166, pp. 310-336 (2005)

17. Paolo, R.: Dynamic Control Card in a Production System Controlled by Conwip Approach. Journal of Mechanical and Industrial Engineering. Vol. 4, Number 4, pp. 425-432 (2010)

18. Sagar, M., Subhash, R.: Development of kanban system for valve manufacturing industries using Just in time principle. IJRASET, Vol. 3, Issue III, pp. 294-298 (2015) 
19. Gamberini, R., Meli, M., Galloni, L., Rimini, B., Lolli, F.: Alternative refilling policies for an assembly line managed by kanbans. Management, and Control International Federation of Automatic Control, pp. 1914-1919 (2013)

20. Qi, H., Weiming, S.: Implementing a hybrid simulation model for a Kanban-based material handling system. Robotics and Computer-Integrated Manufacturing, pp. 635-646 (2008)

21. Jagjit, K.: System dynamics modelling and analysis of just-in time manufacturing systems. University of Windsor, Electronic Theses and Dissertations, Paper 3713 (1991)

22. Alvin, A.: A performance comparison of single product kanban control systems. International Journal of Production Management and Engineering, pp. 57-74 (2014)

23. Chukwunonyelum, E.: Effect of product mix on multi-product pull control. Simulation Modelling Practice and Theory 56, pp. 16-35 (2015)

24. Graves, R., Konopka, J.M., Milne, R.J.: Literature review of material flow control mechanisms. Production Planning and Control 6 (5), pp. 395-403 (1995)

25. Shaojun, W., Bhaba, S.: Production, Manufacturing and Logistics Optimal models for a multi stage supply chain system controlled by kanban under just-in-time philosophy. European Journal of Operational Research, vol. 172, pp. 179-200 (2006)

26. Hussein, M. R.: A Review of Kanban - the Japanese Just in Time Production System. Engineering Management International, vol. 4, pp. 143-150 (1987) 